J. DIFFERENTIAL GEOMETRY

49 (1998) 203-204

\title{
A CORRECTION ON "SOME NONDIFFEOMORPHIC HOMEOMORPHIC HOMOGENEOUS 7-MANIFOLDS WITH POSITIVE SECTIONAL CURVATURE"
}

\author{
MATTHIAS KRECK \& STEPHAN STOLZ
}

As was pointed out to us by L. Astey, E. Micha \& G. Pastor the homeomorphism result in [3] Theorem 3.1 is not correct. They have examples [1] of two non-homeomorphic smooth spin manifolds as in Theorem 3.1 with fourth cohomology group of the same order where the invariants $\bar{s}_{i}$ for $1 \leq i \leq 3$ agree. In these examples the invariant $s_{2}$ is different suggesting that the statement has to be modified by requesting instead of the equality of $\bar{s}_{2}$ the equality of $s_{2}$ (recall that $\bar{s}_{2}$ was simply defined as $2 s_{2}$ ). Theorem 3.1 is based on Proposition 3.2 which in [2] was originally only proved in the smooth category. We assumed that the proof also works in the topological category; this is not true and Proposition 3.2 only holds in the smooth category. At the moment we do not have a classification for topological manifolds. As we will explain below, at least for smooth manifolds the invariant $s_{2}$ is a homeomorphism invariant and one can obtain a homeomorphism classification of smooth manifolds from their diffeomorphism classification. A correct formulation of Theorem 3.1 is:

Theorem 1. Let $M$ and $M^{\prime}$ be smooth manifolds of type (D.1) such that $\left|H^{4}(M ; \mathbb{Z})\right|=\left|H^{4}\left(M^{\prime} ; \mathbb{Z}\right)\right|$ which are both spin or both nonspin. Then $M$ is diffeomorphic (homeomorphic) to $M^{\prime}$ if and only if $s_{i}(M)=$ $s_{i}\left(M^{\prime}\right)$ for $i=1,2,3$ (resp. $28 s_{1}(M)=28 s_{1}\left(M^{\prime}\right)$ and $s_{i}(M)=s_{i}\left(M^{\prime}\right)$ for $i=2,3)$.

Note that the applications to the homeomorphism and diffeomorphism classification of the Wallach spaces is not affected by the mistake

Received October 15, 1996, and, in revised form, March 6, 1996. 
since by Lemmas 4.4 and 5.1 the condition $\bar{s}_{i}(M)=\bar{s}_{i}\left(M^{\prime}\right)$ for $i=1,2,3$ is equivalent to the condition $28 s_{1}(M)=28 s_{1}\left(M^{\prime}\right)$ and $s_{i}(M)=s_{i}\left(M^{\prime}\right)$ for $i=2,3$. This follows since the order of the fourth cohomology group of a Wallach space (denoted $N$ in 4.4 and 5.1) is always odd.

Now we want to explain why $s_{2}$ is a homeomorphism invariant for smooth spin manifolds $M$ of the type under consideration. Let $W$ be compact topological spin manifold whose boundary is $M$ such that the class $u \in H^{2}(M ; \mathbb{Z})$ extends to a class $z \in H^{2}(W ; \mathbb{Z})$. Since $M$ is smooth, the first obstruction for a lift of the topological normal bundle of $W$ to a linear bundle is an element $K S(W) \in H^{4}(W, M ; \mathbb{Z} / 2)$. We say that $W$ is admissible for the computation of $s_{i}$ if $z^{2} \cup K S(W)=0 \in \mathbb{Z} / 2$.

This follows from the fact that both invariants vanish on smooth manifolds and depend only on the class of $(W, z)$ in the reduced bordism group $\tilde{\Omega}_{8}^{\text {TopSpin }}\left(\mathbb{C P}^{\infty}\right)$ of topological spin manifolds. Moreover, both invariants are non-trivial (cf. [3], Lemma 6.2) and the cokernel of the forgetful homeomorphism $\tilde{\Omega}_{8}^{\text {Spin }}\left(\mathbb{C P}^{\infty}\right) \rightarrow \tilde{\Omega}_{8}^{\text {TopSpin }}\left(\mathbb{C P}^{\infty}\right)$ is $Z / 2$; this follows by comparing the Atiyah-Hirzebruch spectral sequences as in [3], Section 6.

As a consequence one can assume, after perhaps adding a closed topological manifold, that for given $M$ the bounding manifold $W$ is admissible. Using only admissible $W$ 's one obtains that $s_{2}(M) \bmod \mathbb{Z}$ is also in the spin case a well defined homeomorphism invariant.

The proof of the theorem above for the homeomorphism classification is reduced to the smooth case by noting that if $\bar{s}_{1}(M)=\bar{s}_{1}\left(M^{\prime}\right)$ then after adding an appropriate homotopy sphere one can assume that $s_{1}(M)=s_{2}\left(M^{\prime}\right)$.

\title{
References
}

[1] L. Astey, E. Micha \& G. Pastor, Homeomorphism and Diffeomorphism types of Eschenburg Spaces, Differential Geom. Appl. 7 (1997) 41-50.

[2] M. Kreck, Surgery and Duality, Preprint, 1996.

[3] M. Kreck \& S. Stolz, Some nondiffeomorphic homeomorphic homogeneous 7-manifolds with positive sectional curvature, Differential Geom. 33 (1991) 465-486.

\author{
Universität Mainz and Mathematisches Forschungsinstitut \\ Oberwolfach, Germany \\ University of Notre Dame, Indiana
}

